\title{
Hematoma Evacuation via Image-Guided Para- Corticospinal Tract Approach in Patients with Spontaneous Intracerebral Hemorrhage
}

\author{
Chao Zhang $\cdot$ Hongfei Ge $\cdot$ Shuixian Zhang $\cdot$ Dan Liu • \\ Zhouyang Jiang $\cdot$ Chuan Lan · Lan Li · Hua Feng $\cdot$ Rong Hu (D)
}

Received: July 26, 2021 / Accepted: August 25, 2021 / Published online: September 12, 2021

(C) The Author(s) 2021

\begin{abstract}
Introduction: Corticospinal tract injury caused by direct hematoma compression and secondary damage induced from blood toxic substances might influence the outcomes in patients with intracerebral hemorrhage (ICH). This study aimed to evaluate the safety and efficacy of hematoma evacuation via imageguided para-corticospinal tract approach based on the protection of compressed or residual corticospinal tract.

Methods: Seventy-five patients with ICH who underwent the image-guided para-corticospinal tract approach were retrospectively collected into the surgery group. Diffusion tensor imaging or computed tomography angiography was performed to identify the relationship between important white matter tracts and hematoma. The neuronavigation system for the preoperative imaging data loaded was used to identify the location of the burr hole, insertion trajectory, and depth of insertion. Cortical entry
\end{abstract}

C. Zhang $\cdot$ H. Ge $\cdot$ S. Zhang $\cdot$ D. Liu $\cdot$ Z. Jiang ·

C. Lan · L. Li · H. Feng ( $₫) \cdot$ R. Hu ( $\square)$

Department of Neurosurgery and Key Laboratory of

Neurotrauma, Southwest Hospital, Third Military

Medical University (Army Medical University),

Chongqing 400038, China

e-mail: fenghua8888@vip.163.com

R. $\mathrm{Hu}$

e-mail: huchrong@tmmu.edu.cn points and insertion trajectories were kept parallel to the corticospinal tract route into the hematoma based on the protection of compressed or residual corticospinal tract. Hematoma was removed under the image-guided para-corticospinal tract approach. Seventy-five age-, sex-, hematoma site-, and volume-matched patients with ICH who underwent conservative treatment were selected as controls. Demographical, clinical, radiological, and treatment-related data were retrospectively analyzed. Functional outcome was evaluated by modified Rankin scale on day 90.

Results: A total of 150 patients with ICH were retrospectively enrolled. The median Glasgow coma scale (GCS) score on admission was 11 (IQR 8-13). Deep hematoma (thalamus and basal ganglion) was present in 86.7\% (130 patients). The mean hematoma volume on admission was $47 \pm 19 \mathrm{~mL}$, and the postoperative hematoma volume was $11 \pm 10 \mathrm{~mL}$. A higher proportion of favorable outcome was observed in the surgery group than in conservative treatment group $(32.0 \%$ versus $17.4 \%$; $p=0.037)$.

Conclusion: Hematoma evacuation via imageguided para-corticospinal tract approach based on the protection of compressed or residual corticospinal tract seems to be safer in patients with ICH with a relatively higher functional independence. 
Keywords: Minimally invasive; Paracorticospinal tract; Hematoma evacuation; Surgery; Intracerebral hemorrhage

\section{Key Summary Points}

\section{Why carry out this study?}

The most common site of intracerebral hemorrhage (ICH) (approximately 80\%) is the basal ganglia, and corticospinal tract (CST) is the main descending fiber bundle of posterior limbs of internal capsule that pivotally maintains motor function. Hence, it is important to explore methods for protecting the compressed or remaining CST after ICH.

The aim of the study was to evaluate the safety and efficacy of hematoma evacuation via image-guided paracorticospinal tract approach based on the protection of compressed or residual corticospinal tract. To date, there are fewer studies that evaluate the safety and efficacy of the approach.

\section{What was learned from the study?}

It suggests that the hematoma evacuation via image-guided para-corticospinal tract approach based on the protection of compressed or residual corticospinal tract seems to be safe in patients with ICH with a relatively higher functional independence.

Additionally, the pragmatic use of hematoma evacuation via image-guided para-corticospinal tract approach could serve as a recommended therapeutic method for patients with ICH.

\section{INTRODUCTION}

Spontaneous intracerebral hemorrhage (ICH) remains the poorest prognosis subtype of stroke [1], with independency rates of between $12 \%$ 


\section{METHODS}

\section{Patients}

Ethics approval was obtained from the Ethics Committee of the Southwest Hospital of Third Military Medical University (approval no. 2017niankeyandi58hao). A consecutive series of 75 patients with spontaneous ICH underwent hematoma evacuation via image-guided paraCST approach was retrospectively collected into the surgery group from January 7, 2013 to November 14, 2020. Candidates in the conservative treatment group were selected from the patients whose legal guardian refused to allow surgical treatment after informed consent. Age ( \pm 5 years)-, sex-, hematoma site-, and volume $( \pm 5 \mathrm{~mL}$ )-matched patients with $\mathrm{ICH}$ who underwent conservative treatment were selected as controls (1:1 match). Eligible patients with ICH with a stable supratentorial volume of greater than $25 \mathrm{~mL}$ calculated by two experienced doctors, with minimal or no intraventricular extension, were included. Patients with Glasgow coma scale (GCS) score $\leq 4$, apparent signs of brain herniation (bilateral fixed, dilated pupils, etc.), infratentorial hemorrhage, aneurysm, vascular malformation, tumor, head trauma, or anticoagulant-associated pathogenesis were restricted from enrollment. Pregnant women were also excluded from this study.

All patients were admitted to a neurosurgical intensive care unit, and initiated early management according to guidelines [21]. In order to reduce the risk of hematoma expansion or postoperative rebleeding, systolic blood pressure was maintained at less than $140 \mathrm{mmHg}$. Patients received physiotherapy from admission to discharge, and rehabilitative treatments were organized for each patient according to the neurologic function after discharge [22].

\section{Clinical Data}

Medical records were examined, and patients or their family members were interviewed to collect clinical data. Demographic data included sex, age, smoking history, drinking history, and medical history (hypertension, diabetes mellitus, coronary artery disease, and history of stroke). Clinical features on admission including blood pressure, GCS score, National Institute of Health stroke scale (NIHSS) score, and time to admission were also analyzed. Radiological data including ICH volumes (hematoma volume measured via semi-automated planimetry method) [14], hematoma location, and intraventricular hemorrhage were determined by two experienced doctors who were blinded to the research. The surgical data comprised time to surgery, bleeding during surgery, time of surgery, clot clearance rate, and reoperation. Treatment-related data (the intracranial pressure [ICP; recorded every $2 \mathrm{~h}$ for 5 days] and complications [pneumonia, hydrocephalus, gastrointestinal bleeding, hematoma expansion, intracranial infection, seizures]) was selected according to the medical records. Functional outcome, performed by a designated neurologist who was blinded to the primary outcome, was evaluated on day 90. Follow-up was performed by a face-to-face interview or telephone call. The functional outcome was measured by modified Rankin scale (mRS) [14]. Poor outcomes were defined as mRS grade 4-6 (mRS $4=$ moderately severe disability; mRS $6=$ dead).

\section{Surgical Procedures}

For all patients that received surgery, diffusion tensor imaging (DTI; repetition time, $5000 \mathrm{~ms}$; echo time, $92 \mathrm{~ms}$; motion probing gradient (MPG), 30 axes; $b$ value, $0,1000 \mathrm{~s} / \mathrm{mm}^{2}$; matrix, $128 \times 128$; number of excitations (NEX), 1 ; slice thickness/interleaved, $4 \mathrm{~mm}$ ) was performed using a 3-T magnetic resonance image (MRI) system (SIEMENS Magnetom Trio a Tim 3.0 T, Erlangen, Germany) if the conditions allowed after acquiring computed tomographic angiography (CTA) scans. If an MRI was not feasible because of magnetic substances in patients with ICH or because the MRI scan might delay appropriate management, trajectory planning was abandoned. CTA $(120 \mathrm{kVp}$; $600 \mathrm{~mA}$; section thickness, $0.625 \mathrm{~mm}$ ) was performed with a 128-slice multidetector CT scanner (SIEMENS Somatom Volume Zoom, 
Erlangen, Germany). A total of $80 \mathrm{~mL}$ of nonionic contrast medium (Visipaque 320; GE Healthcare) was injected through the antecubital vein at a rate of $4-4.5 \mathrm{~mL} / \mathrm{s}$. The location of important white matter tracts can be inferred from the CT on the basis of the relationship between hematoma and CST when necessary [23-25].

Under general anesthesia, the patient was placed in a supine position and the head was fixed with a Mayfield headholder. Preoperative imaging data were loaded into the neuronavigation system (StealthStation7 Navigation System, Medtronic Inc., Minneapolis, MN, USA). A $3 \mathrm{D}$ reconstruction of the hematoma using this system was determined using the CT or MRI scans to design the location of the burr hole, insertion trajectory, and depth of insertion during the operative procedures. Cortical entry points and insertion trajectories were kept parallel to the CST route into the hematoma based on the protection of compressed or residual corticospinal tract (Fig. 1). After the scalp was shaved and prepared in the routine sterile fashion, cranial landmarks were registered with the Stealthstation7 navigation system. Then, an incision was made in the scalp, consistent with the planned surgery trajectory. An ipsilateral frontal hole was created. Subsequently, the dura was opened, and the pia was dissected along the sulcus of interest. Endoscopic or microscopic surgery, depending on the availability of neuroendoscope or microscope, was introduced into the sulcus under navigation guidance and parallel to the CST into the hematoma along the trajectory. To ensure the accuracy, the surgeon should navigate from the entry point to the target by viewing axial, coronal, and sagittal images simultaneously to ensure that the navigating tool was on target in all three planes. Intracranial pressure (ICP) is measured by use of an ICP monitoring device (Codman, Johnson and Johnson Medical Ltd, Raynhan, MA) inserted into the brain parenchyma or cerebral ventricles.

\section{Statistical Analysis}

Demographical, clinical, radiological, and treatment-related data of all enrolled patients were retrospectively collected. Continuous variables were analyzed using Student's $t$ test or nonparametric test. Categorical variables were analyzed using the chi-square $\left(\chi^{2}\right)$ test or
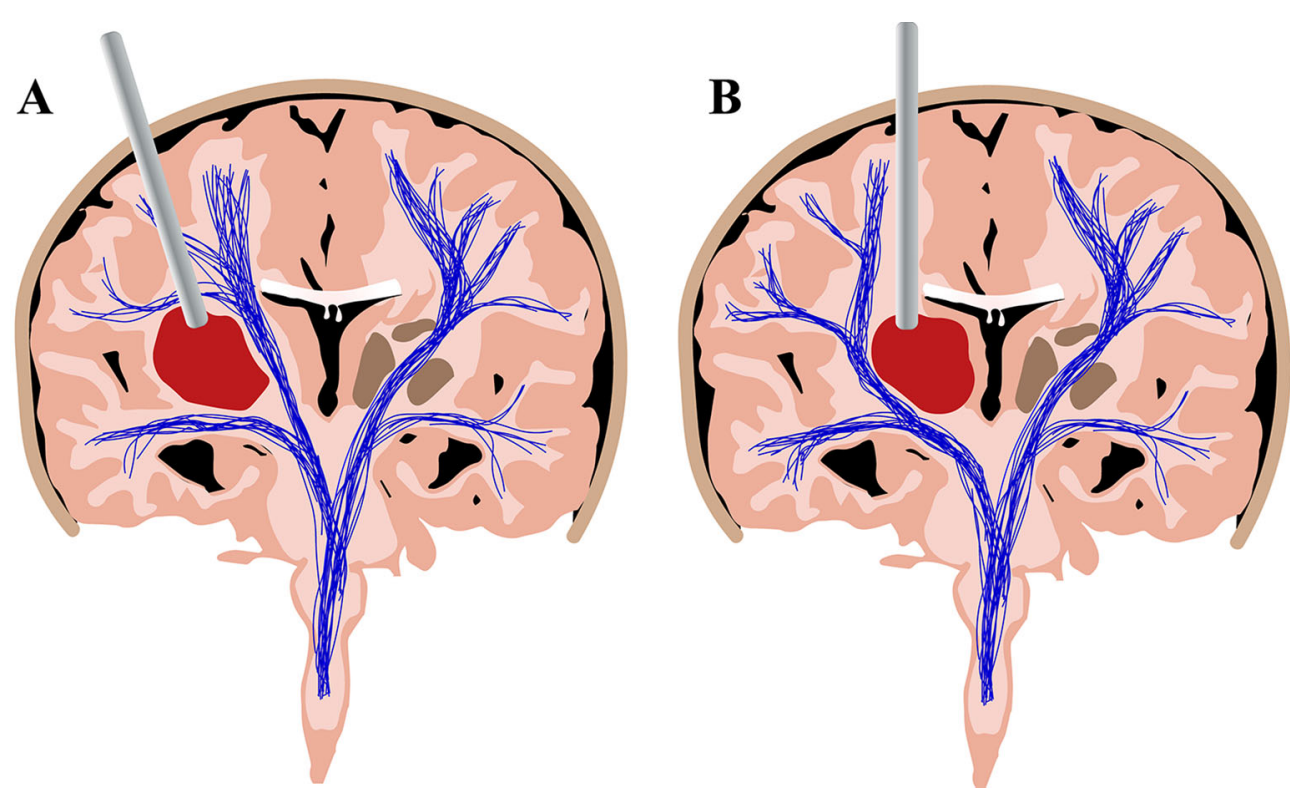

Fig. 1 Illustration of the para-CST approach for hematoma evacuation when the CST is located a outside or $\mathbf{b}$ inside the hematoma. CST corticospinal tract 
Table 1 Demographical data of the 150 patients with ICH

\begin{tabular}{|c|c|c|c|}
\hline & Surgery group $(n=75)$ & Conservative treatment group $(n=75)$ & $p$ \\
\hline Age, years, mean $\pm S D$ & $58 \pm 13$ & $58 \pm 13$ & 0.970 \\
\hline Male, $n(\%)$ & $57(76.0)$ & $57(76.0)$ & 1.000 \\
\hline Time to admission, h, median (IQR) & $10(6,24)$ & $10(6,24)$ & 0.687 \\
\hline \multicolumn{4}{|l|}{ Medical history, $n$ (\%) } \\
\hline Hypertension & $55(73.3)$ & $64(85.3)$ & 0.070 \\
\hline Diabetes mellitus & $5(6.7)$ & $11(14.7)$ & 0.113 \\
\hline Coronary artery disease & $4(5.3)$ & $8(10.7)$ & 0.229 \\
\hline History of stroke & $16(21.3)$ & $16(21.3)$ & 1.000 \\
\hline Smoking & $26(34.7)$ & $16(21.3)$ & 0.069 \\
\hline Drinking & $24(32.0)$ & $31(1.3)$ & 0.236 \\
\hline \multicolumn{4}{|l|}{ Clinical features } \\
\hline Systolic BP, mmHg, mean \pm SD & $173 \pm 27$ & $173 \pm 29$ & 0.986 \\
\hline Diastolic BP, mmHg, mean $\pm \mathrm{SD}$ & $94 \pm 15$ & $97 \pm 16$ & 0.227 \\
\hline GCS score, median (IQR) & $11(8,13)$ & $11(8,13)$ & 0.792 \\
\hline NIHSS score, mean $\pm S D$ & $14 \pm 7$ & $14 \pm 7$ & 0.741 \\
\hline \multicolumn{4}{|l|}{ Characteristics of hematomas } \\
\hline Site, $n(\%)$ & & & 1.000 \\
\hline Deep & $65(86.7)$ & $65(86.7)$ & \\
\hline Lobar & $10(13.3)$ & $10(13.3)$ & \\
\hline Volume, $\mathrm{mL}$, mean $\pm \mathrm{SD}$ & $47 \pm 17$ & $47 \pm 18$ & 0.835 \\
\hline Extension to ventricles, $n(\%)$ & $27(36.0)$ & $21(28.0)$ & 0.294 \\
\hline
\end{tabular}

$I C H$ intracerebral hemorrhage, IQR Interquartile range, $S D$ standard deviation, $B P$ blood pressure, GCS Glasgow coma scale, NIHSS National Institute of Health stroke scale

continuity correction test. Significant variables $(p<0.2)$ were entered into the multivariable analysis (binary logistic regression model) to evaluate factors for favorable outcome of patients with ICH. Pearson test was used to analyze the correlation between the postoperative hematoma volume and NIHSS score at 3 months. All statistical tests were two-sided, and a $p<0.05$ was considered to be statistical significant. Statistical analyses were performed using SPSS software for Windows (version 22.0, Inc., Chicago, IL).

\section{RESULTS}

\section{Baseline Characteristics}

Seventy-five patients with ICH who underwent the image-guided para-corticospinal tract approach were retrospectively collected into the surgery group between January 7, 2013 and November 14, 2020. The patients in the conservative treatment group comprised 75 age-, sex-, hematoma site-, and volume-matched 
Table 2 Surgical data of the 75 patients with $\mathrm{ICH}$

\begin{tabular}{|c|c|}
\hline & Surgery group $(n=75)$ \\
\hline \multicolumn{2}{|l|}{ Treatment variables } \\
\hline Time to surgery, h, median (IQR) & $21(13,40)$ \\
\hline Surgery within $12 \mathrm{~h}$ from ictus, $n(\%)$ & $15(20.0)$ \\
\hline Surgery within $24 \mathrm{~h}$ from ictus, $n(\%)$ & $43(57.3)$ \\
\hline Bleeding during surgery, $\mathrm{mL}$, mean $\pm \mathrm{SD}$ & $308 \pm 208$ \\
\hline Time of surgery, h, mean $\pm S D$ & $3 \pm 2$ \\
\hline External ventricular drain, $n(\%)$ & $5(6.7)$ \\
\hline ICP events monitored, $n(\%)$ & $64(85.3)$ \\
\hline Postoperative $\mathrm{ICH}$ volume, $\mathrm{mL}$, mean $\pm \mathrm{SD}$ & $11 \pm 10$ \\
\hline Postoperative $\mathrm{ICH}$ volume $\leq 15 \mathrm{~mL}, n(\%)$ & $52(69.3)$ \\
\hline \multicolumn{2}{|l|}{ ICP of postoperative, $\mathrm{mmHg}$, mean $\pm \mathrm{SD}$} \\
\hline Intraoperative & $7 \pm 4$ \\
\hline Day 1 & $10 \pm 5$ \\
\hline Day 2 & $12 \pm 5$ \\
\hline Day 3 & $12 \pm 4$ \\
\hline Day 4 & $11 \pm 4$ \\
\hline Day 5 & $10 \pm 4$ \\
\hline Any ICP events $\geq 20 \mathrm{~mm} \mathrm{Hg}, n(\%)$ & $20(31.3)$ \\
\hline Any ICP events $\geq 30 \mathrm{~mm} \mathrm{Hg}, n(\%)$ & $3(4.7)$ \\
\hline Reoperation, $n$ (\%) & $4(5.3)$ \\
\hline
\end{tabular}

$I C H$ intracerebral hemorrhage, $I Q R$ interquartile range, $S D$ standard deviation, ICP intracranial pressure

candidates that were selected from the conservative therapeutic patients. The mean age was $59 \pm 13$ years, and $114(76.0 \%)$ patients were male. The GCS score on admission was 11 (8, 13). The NIHSS score on admission was $14 \pm 7$. The mean hematoma volume was $47 \pm 19 \mathrm{~mL}$, and $130(86.7 \%)$ patients had a deep hematoma (Table 1).

\section{Treatment}

Neuronavigation was used in $100 \%$ of the cases. A total of 22 patients received MRI scan, and 53 patients received CT scan before surgery. The postoperative volume of hematoma was $11 \pm 10 \mathrm{~mL}$. The postoperative ICH volume was not associated with functional outcome $(p=0.094)$, and the outcome in the group of postoperative ICH volume less than $15 \mathrm{~mL}$ was not better than that of postoperative ICH volume more than $15 \mathrm{~mL}$ at 3 months $(p=0.815)$. The ICP data was available during 5 days after operation. The mean ICP was less than $15 \mathrm{mmHg}$ every day and the peak of mean ICP was $12 \mathrm{mmHg}$ on day 2. Four (5.3\%) patients needed reoperation because of brain edema and symptomatic brain bleeding after surgery (Table 2). 
Table 3 Outcomes and complications

\begin{tabular}{|c|c|c|c|}
\hline & Surgery group $(n=75)$ & Conservative treatment group $(n=75)$ & $p$ \\
\hline \multicolumn{4}{|l|}{ Primary outcome } \\
\hline Favorable $(\mathrm{mRS}=1-3), n(\%)$ & $24(32.0)$ & $13(17.4)$ & 0.037 \\
\hline Unfavorable $(\mathrm{mRS}=4-6), n(\%)$ & $51(68.0)$ & $62(82.6)$ & \\
\hline \multicolumn{4}{|l|}{ Secondary outcomes } \\
\hline Died within $0-90$ days, $n$ (\%) & $11(14.7)$ & $19(25.3)$ & 0.102 \\
\hline Modified Rankin scale, $n(\%)$ & & & 0.056 \\
\hline 1 & $1(1.3)$ & $3(4.0)$ & \\
\hline 2 & $7(9.3)$ & $6(8.0)$ & \\
\hline 3 & $16(21.4)$ & $4(5.3)$ & \\
\hline 4 & $37(49.3)$ & $41(54.7)$ & \\
\hline 5 & $3(4.0)$ & $2(2.7)$ & \\
\hline 6 & $11(14.7)$ & $19(25.3)$ & \\
\hline NIHSS score at 90 days, mean \pm SD & $14 \pm 12$ & $18 \pm 15$ & 0.029 \\
\hline Time in hospital, days, mean \pm SD & $27 \pm 19$ & $22 \pm 28$ & 0.292 \\
\hline Time in ICU, days, mean \pm SD & $12 \pm 9$ & $8 \pm 18$ & 0.119 \\
\hline \multicolumn{4}{|l|}{ Complications, $n(\%)$} \\
\hline Hydrocephalus & $4(5.3)$ & $5(6.7)$ & 1.000 \\
\hline Pneumonia & $61(81.3)$ & $54(72.0)$ & 0.177 \\
\hline GI bleeding & $7(9.3)$ & $6(8.0)$ & 0.772 \\
\hline Hematoma expansion & $18(24.0)$ & $13(17.3)$ & 0.313 \\
\hline Intracranial infection & $2(2.7)$ & $0(0.0)$ & 0.477 \\
\hline Seizures & $5(6.7)$ & $1(1.3)$ & 0.211 \\
\hline
\end{tabular}

$I C H$ intracerebral hemorrhage, $m R S$ modified Rankin scale, IQR interquartile range, $S D$ standard deviation, NIHSS National Institute of Health stroke scale, $I C U$ intensive care unit, GI gastrointestinal

\section{Outcomes}

The modified Rankin scale (mRS) score at the last follow-up was available for all patients. No patients were lost on day 90 after surgery. A total of $24(32.0 \%)$ of 75 patients in the surgery group and $13(17.4 \%)$ of 75 patients in the conservative treatment group had an mRS score of 3 or less at 90 days after ICH $(p=0.037)$. The surgery group exhibited a lower death rate, but there was no significant difference $(14.7 \%$ versus $25.3 \% ; p=0.102$ ) (Table 3 ). Among them, 115 (76.7\%) patients suffered from pneumonia, which was the major complication. The rates of occurrence of other complications such as hydrocephalus, hematoma expansion, intracranial infection, and seizures in the surgery group were $5.3 \%, 24.0 \%, 2.7 \%$, and $6.7 \%$, respectively (Table 3). 
Table 4 Characteristics and outcomes of patients who underwent MRI- or CT-guided para-CST approach

\begin{tabular}{|c|c|c|c|}
\hline & MRI group $(n=22)$ & CT group $(n=53)$ & $p$ \\
\hline Age, years, mean $\pm S D$ & $51 \pm 10$ & $61 \pm 13$ & 0.002 \\
\hline Male, $n(\%)$ & $17(77.3)$ & $40(75.5)$ & 0.868 \\
\hline Time to imaging scan, $h$, median (IQR) & $25(11,59)$ & $9(5,19)$ & 0.000 \\
\hline Site, $n(\%)$ & & & 0.242 \\
\hline Deep & $17(77.3)$ & $48(90.6)$ & \\
\hline Lobar & $5(22.7)$ & $5(9.4)$ & \\
\hline Volume, $\mathrm{mL}$, mean $\pm \mathrm{SD}$ & $40 \pm 11$ & $50 \pm 18$ & 0.003 \\
\hline \multicolumn{4}{|l|}{ Surgical data } \\
\hline Postoperative $\mathrm{ICH}$ volume, $\mathrm{mL}$, mean $\pm \mathrm{SD}$ & $8 \pm 8$ & $12 \pm 11$ & 0.056 \\
\hline Reoperation, $n$ (\%) & $3(13.6)$ & $1(1.9)$ & 0.134 \\
\hline \multicolumn{4}{|l|}{ Outcome } \\
\hline Favorable $(\mathrm{mRS}=1-3), n(\%)$ & $11(50.0)$ & $13(24.5)$ & 0.031 \\
\hline Unfavorable $(\mathrm{mRS}=4-6), n(\%)$ & $11(50.0)$ & $40(75.5)$ & \\
\hline Died within 0-90 days, $n$ (\%) & $3(13.6)$ & $8(15.1)$ & 0.779 \\
\hline NIHSS score at 90 days, mean \pm SD & $11 \pm 13$ & $15 \pm 12$ & 0.233 \\
\hline \multicolumn{4}{|l|}{ Complications, $n$ (\%) } \\
\hline Hydrocephalus & $0(0.0)$ & $4(7.5)$ & 0.447 \\
\hline Pneumonia & $15(68.2)$ & $47(88.7)$ & 0.072 \\
\hline Intracranial infection & $0(0.0)$ & $2(3.8)$ & 0.891 \\
\hline Seizures & $2(9.1)$ & $3(5.7)$ & 0.973 \\
\hline
\end{tabular}

$I C H$ intracerebral hemorrhage, $m R S$ modified Rankin scale, IQR interquartile range, $S D$ standard deviation, NIHSS National Institute of Health stroke scale, $C T$ computed tomography, MRI magnetic resonance imaging

\section{Outcomes in Patients Who Underwent MRI- or CT-Guided Para-CST Approach}

Given that CT is a widely and rapidly available technique for patients with $\mathrm{ICH}$, the question arises whether the CT-guided para-CST approach could be used instead of MRI-guided para-CST approach in clinical practice. To assess and compare the safety and efficacy between CT-guided para-CST approach and MRI-guided para-CST method, we analyzed the characteristics and outcomes of patients who underwent MRI- or CT-guided para-CST approach. The results demonstrated that patients who underwent CT-guided para-CST approach had higher age and a greater volume of hematoma. There was no overt difference between hematoma sites (Table 4). Compared to patients who underwent CT-guided para-CST approach, the proportion of patients with unfavorable functional outcome was significantly decreased in patients who underwent MRI-guided para-CST approach at 3 months [mRS score $3-6$ at 3 months was $13 / 53(24.5 \%)$ for CT vs $11 / 22$ (50.0\%) for MRI; $p=0.031]$. 

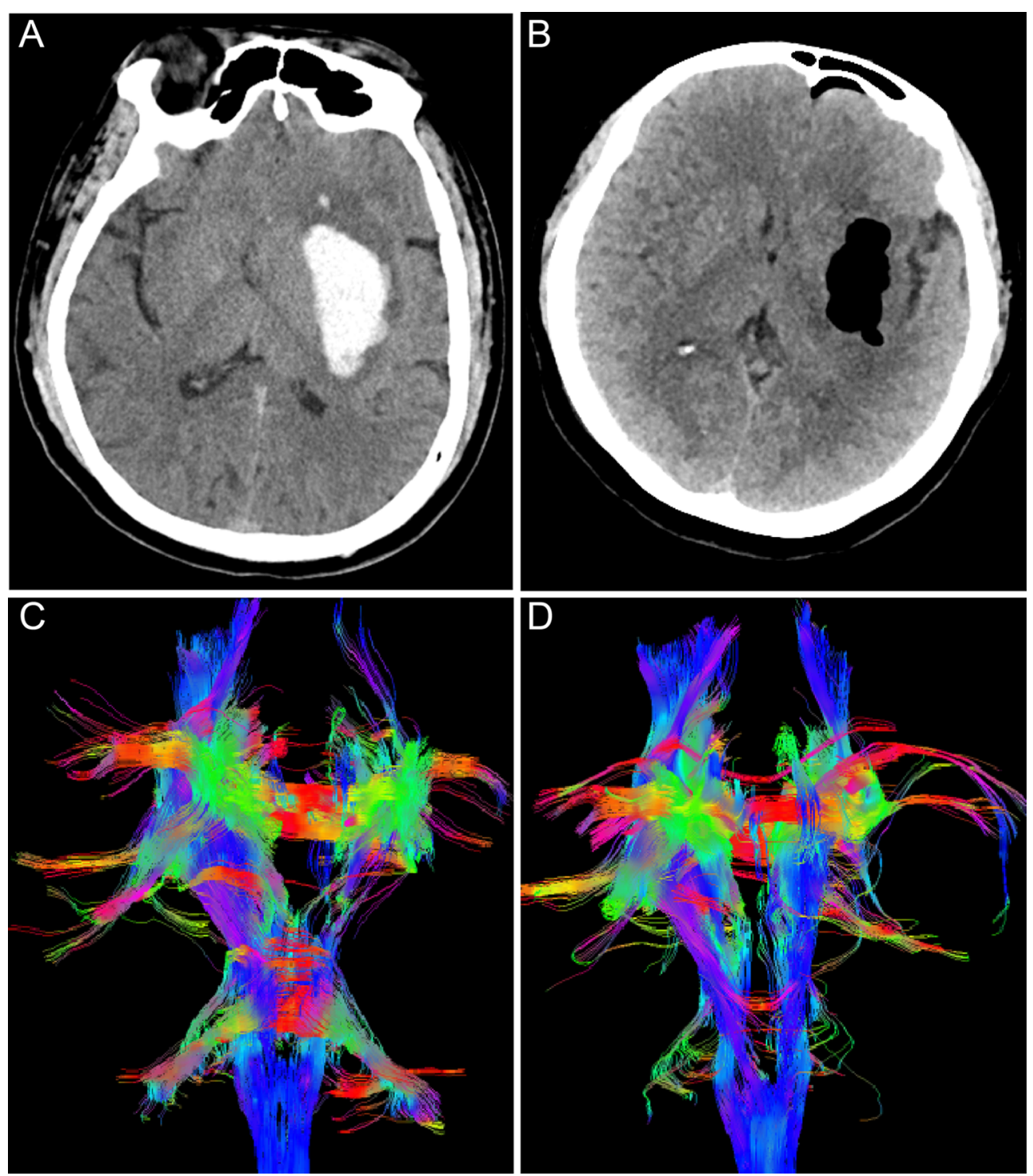

Fig. 2 Illustrative case of basal ganglia ICH in a previously healthy 47-year-old woman. a Preoperative axial CT scan without contrast. b Postoperative CT scan showing complete hematoma evacuation. c Preoperative axial DTI scan showing that the corticospinal tract was partially

interrupted. d Postoperative DTI scan showing the recovery of a partially interrupted corticospinal tract. $\mathrm{ICH}$ intracerebral hemorrhage, DTI diffusion tensor imaging

\section{Predictors of Favorable Outcome for Patients with ICH}

Multivariate logistic regression analyses showed that lower hematoma $(p=0.005)$, lower NIHSS score $(p=0.000)$, lobar hemorrhage $(p=0.012)$, and history of stroke $(p=0.004)$ were

independent predictors of favorable outcome for patients with ICH. Hematoma evacuation via image-guided para-corticospinal tract approach was found to be a significant predictor of favorable outcome for patients with ICH (OR $5.237,95 \%$ CI $1.595-17.199, p=0.006$ ). 


\section{Illustrative Cases}

A 47-year-old woman suffered from right limb dysfunction. Basal ganglia intracerebral hemorrhage was detected on head CT scan (Fig. 2a). The hematoma was completely evacuated (Fig. 2b). The corticospinal tract was partially interrupted on the DTI scan before surgery (Fig. 2c). On day 90 post-injury, the patient recovered and achieved an mRS score of 3 . Follow-up DTI on day 180 showed the recovery of a partially interrupted corticospinal tract (Fig. 2d).

\section{DISCUSSION}

ICH is a deadly disease which affects 2 million people worldwide each year $[1,26]$. The world's annual mortality rate is as high as $40-50 \%$, with $60-80 \%$ of survivors suffering from residual neurological dysfunction [2]. Thus far, no effective treatments are available for patients with $\mathrm{ICH}[2,5]$. The role of surgery in treating patients with $\mathrm{ICH}$ still remains controversial $[3,4]$. Despite the progress of technology, early surgery does not show better prognosis when compared with initial conservative treatment $[5,6]$. In the STICH II trial, the mortality rate is $18 \%$ and the disability rate is $38 \%$ when the hematoma is located in the cortex [6]. Iatrogenic insults from clot evacuation via open craniotomy might be a reason for the unsatisfactory prognosis $[7,8]$. Meanwhile, minimally invasive surgery for the treatment of ICH has been demonstrated to be a safe therapeutic strategy in improving functional outcomes, compared with conventional craniotomy $[9,10,12,27-29]$. These methods include endoscopic removal, stereotactic evacuation, or the injection of thrombolysis for clot dissolution $[13,27]$. However, aspiration and thrombolytic irrigation of the hematoma with alteplase via an image-guided catheter did not improve functional outcomes, compared to standard medical care in patients with large intracerebral hemorrhage in the MISTIE III trial [14]. The mortality and disability rates still remain high, $19 \%$ and $36 \%$, respectively [14]. The present study showed the mortality rate was $14.7 \%$ and the disability rate was $53.3 \%$. The reason for high disability was that the rate of death decreased along with the rate of increasing disability, and the bleeding site was mostly located in the deep brain. The rate of deep bleeding was $65 \%$ in the MISTIE group of the MISTIE III trial [14]. In our study, the rate of deep bleeding was $86.7 \%$. The mortality was lower than that in the STICH I (37\%), STICH II (21\%), and MISTIE III (19\%) [5, 6, 14]. This result showed that the treatment of ICH by para-CST approach had some advantages, compared with craniotomy and minimally invasive surgery with thrombolysis.

CST integrity is a decisive factor in motor recovery, and CST preservation is important for motor recovery [15-17]. We found that a clear difference between CST preservation and CST interruption groups in terms of NIHSS score at 3 months [7 (IQR 3-10) vs 10 (IQR 10-14); $p=0.021$ [ [30]. Therefore, the approach is crucial for the degree of fiber bundle damage and postoperative motor function, especially for deep hematomas. Traditional surgical methods often choose the shortest path to the hematoma, such as the use of temporal lobe or frontal lobe lateral cortex fistula, so the operation itself may cause secondary fiber bundle damage. Some studies show that minimally invasive surgery for hematoma evacuation could reduce the damage to CST $[18,20,27]$. The para-CST approach protects the fiber bundle to the greatest extent to avoid iatrogenic injury. Patients who underwent hematoma evacuation via image-guided para-CST approach exhibited a better outcome than patients who underwent conservative treatment group $(p=0.037)$. Two reasons might contribute to the preservation of compressed or residual corticospinal tract in this approach. Firstly, the preoperative DTI clarifies the direction of the fiber bundles and the relationship between hematoma and fibers, which is beneficial for protecting the compressed or residual corticospinal tract. Preoperative CTA scan identifies the important blood vessels to reduce the risk of vessels injury. Secondly, intraoperative navigation guides the best suitable path, which assists evacuation avenue parallel to the CST entering into the hematoma. Therefore, hematoma evacuation via image- 
guided para-CST approach is a potential strategy for the treatment of patients with ICH. However, there are also some disadvantages. Only 22 patients received DTI scan because it was not feasible or it would have delayed appropriate management, and hence the CT scan was used to navigate this condition [23-25, 31]. Patients who underwent MRI-guided para-CST approach had a better outcome than patients who underwent CT-guided para-CST approach $(p=0.031)$, but there is no difference between mortality $(p=0.779)$. Some factors may have contributed to this result. Patients who underwent CT-guided para-CST approach had higher age and a greater volume of hematoma $(p<0.05)$. There may be bias because of the small number of cases and the characteristics of this retrospective study.

Our approach also delineates a lower rate of complications due to surgery. The rates of reoperation, intracranial infection, and hydrocephalus were $5.3 \%, 2.7 \%$, and $5.3 \%$, respectively. The results indicated that the surgical method was safe. A limitation of our study was the small sample size of patients that limits the extension to a broader population. Thus, a prospective, multicenter, randomized trial needs to be conducted to determine the efficacy and safety of the approach.

\section{CONCLUSIONS}

Hematoma evacuation via image-guided paracorticospinal tract approach is safe in our study, with a relatively higher functional independence at 90 days. The approach is an effective method for intracerebral hemorrhage evacuation, which can decrease the iatrogenic insults, compared with craniotomy. The new rational surgical approach through para-corticospinal tract using neuronavigation guidance is reliable and logical in protecting compressed or residual corticospinal tract. Therefore, hematoma evacuation via image-guided para-corticospinal tract approach seems to be safer in patients with ICH with a relatively higher functional independence, and it could be a promising approach for $\mathrm{ICH}$ in the future.

\section{ACKNOWLEDGEMENTS}

The authors would like to say thanks for all the patients, their families, and all investigators involved in this study.

Funding. This work was supported by grants from the Program for Innovation Research Group of Universities in Chongqing (No. 2019cqspt15), Collaborative Innovation Center for Brain Science of Chongqing (NO. 4174D7) and Talent Project of Army Medical University (No. XZ-2019-505-024). The journal's Rapid Service Fee was funded by the authors.

Authorship. All named authors meet the International Committee of Medical Journal Editors (ICMJE) criteria for authorship in this article, take responsibility for the integrity of the work as a whole, and have given their approval for this version to be published.

Authors' Contributions. Study concept and design: RH and HF; Data collection: CZ, HFG, SXZ, DL, ZYJ, CL, and LL. Statistical analysis: RH and CZ. Drafting of the manuscript: RH and HF. All authors revised the manuscript and approved the final version to be published.

Disclosures. Chao Zhang, Hongfei Ge, Shuixian Zhang, Dan Liu, Zhouyang Jiang, Chuan Lan, Lan Li, Hua Feng, and Rong $\mathrm{Hu}$ have nothing to disclose.

Compliance with Ethics Guidelines. Ethics approval was obtained from the Ethics Committee of the Southwest Hospital of Third Military Medical University (No. 2017niankeyandi58hao).

Data Availability. All data generated or analyzed during this study are available from the corresponding author on reasonable request.

Open Access. This article is licensed under a Creative Commons Attribution-NonCommercial 4.0 International License, which permits any non-commercial use, sharing, adaptation, 
distribution and reproduction in any medium or format, as long as you give appropriate credit to the original author(s) and the source, provide a link to the Creative Commons licence, and indicate if changes were made. The images or other third party material in this article are included in the article's Creative Commons licence, unless indicated otherwise in a credit line to the material. If material is not included in the article's Creative Commons licence and your intended use is not permitted by statutory regulation or exceeds the permitted use, you will need to obtain permission directly from the copyright holder. To view a copy of this licence, visit http://creativecommons.org/licenses/bync/4.0/.

\section{REFERENCES}

1. Qureshi AI, Mendelow AD, Hanley DF. Intracerebral haemorrhage. Lancet. 2009;373(9675):1632-44.

2. van Asch CJ, Luitse MJ, Rinkel GJ, van der Tweel I, Algra A, Klijn CJ. Incidence, case fatality, and functional outcome of intracerebral haemorrhage over time, according to age, sex, and ethnic origin: a systematic review and meta-analysis. Lancet Neurol. 2010;9(2):167-76.

3. Fernandes HM, Gregson B, Siddique S, Mendelow AD. Surgery in intracerebral hemorrhage. The uncertainty continues. Stroke. 2000;31(10):2511-6.

4. Akhigbe T, Zolnourian A. Role of surgery in the management of patients with supratentorial spontaneous intracerebral hematoma: critical appraisal of evidence. J Clin Neurosci. 2017;39:35-8.

5. Mendelow AD, Gregson BA, Fernandes HM, et al. Early surgery versus initial conservative treatment in patients with spontaneous supratentorial intracerebral haematomas in the International Surgical Trial in Intracerebral Haemorrhage (STICH): a randomised trial. Lancet. 2005;365(9457):387-97.

6. Mendelow AD, Gregson BA, Rowan EN, Murray GD, Gholkar A, Mitchell PM. Early surgery versus initial conservative treatment in patients with spontaneous supratentorial lobar intracerebral haematomas (STICH II): a randomised trial. Lancet. 2013;382(9890):397-408.
7. Frösen J, Jahromi BR, Hernesniemi J. Intracerebral hemorrhage as a surgical challenge-where should we focus? World Neurosurg. 2016;91:638-9.

8. Flaherty ML, Beck J. Surgery for intracerebral hemorrhage: moving forward or making circles? Stroke. 2013;44(10):2953-4.

9. Zuccarello M, Andaluz N, Wagner KR. Minimally invasive therapy for intracerebral hematomas. Neurosurg Clin N Am. 2002;13(3):349-54.

10. Scaggiante J, Zhang X, Mocco J, Kellner CP. Minimally invasive surgery for intracerebral hemorrhage. Stroke. 2018;49(11):2612-20.

11. Nagasaka T, Tsugeno M, Ikeda $H$, Okamoto $T$, Inao S, Wakabayashi T. Early recovery and better evacuation rate in neuroendoscopic surgery for spontaneous intracerebral hemorrhage using a multifunctional cannula: preliminary study in comparison with craniotomy. J Stroke Cerebrovasc Dis. 2011;20(3):208-13.

12. Xia Z, Wu X, Li J, et al. Minimally invasive surgery is superior to conventional craniotomy in patients with spontaneous supratentorial intracerebral hemorrhage: a systematic review and meta-analysis. World Neurosurg. 2018;115:266-73.

13. Hanley DF, Thompson RE, Muschelli J, et al. Safety and efficacy of minimally invasive surgery plus alteplase in intracerebral haemorrhage evacuation (MISTIE): a randomised, controlled, open-label, phase 2 trial. Lancet Neurol. 2016;15(12):1228-37.

14. Hanley DF, Thompson RE, Rosenblum M, et al. Efficacy and safety of minimally invasive surgery with thrombolysis in intracerebral haemorrhage evacuation (MISTIE III): a randomised, controlled, open-label, blinded endpoint phase 3 trial. Lancet. 2019;393(10175):1021-32.

15. Chang MC, Do KH, Chun MH. Prediction of lower limb motor outcomes based on transcranial magnetic stimulation findings in patients with an infarct of the anterior cerebral artery. Somatosens Mot Res. 2015;32(4):249-53.

16. Jang $\mathrm{SH}$, Choi BY, Chang $\mathrm{CH}$, Kim $\mathrm{SH}$, Chang MC. Prediction of motor outcome based on diffusion tensor tractography findings in thalamic hemorrhage. Int J Neurosci. 2013;123(4):233-9.

17. Moon JS, Chung SM, Jang SH, Won KC, Chang MC. Effects of diabetes on motor recovery after cerebral infarct: a diffusion tensor imaging study. J Clin Endocrinol Metab. 2019.

18. Hansen BM, Ullman N, Muschelli J, et al. Relationship of white matter lesions with intracerebral hemorrhage expansion and functional outcome: 
MISTIE II and CLEAR III. Neurocrit Care. 2020;33: $516-24$

19. Jang SH. The role of the corticospinal tract in motor recovery in patients with a stroke: a review. NeuroRehabilitation. 2009;24(3):285-90.

20. Lee AY, Choi BY, Kim SH, Chang CH, Jung YJ, Jang $\mathrm{SH}$. Difference of injury of the corticospinal tract according to surgical or conservative treatment in patients with putaminal hemorrhage. Int J Neurosci. 2016;126(5):429-35.

21. Hemphill JC 3rd, Greenberg SM, Anderson CS, et al. Guidelines for the management of spontaneous intracerebral hemorrhage: a guideline for healthcare professionals from the American Heart Association/American Stroke Association. Stroke. 2015;46(7):2032-60.

22. Winstein CJ, Stein J, Arena R, et al. Guidelines for adult stroke rehabilitation and recovery: a guideline for healthcare professionals from the American Heart Association/American Stroke Association. Stroke. 2016;47(6):e98-169.

23. Kellner CP. Long-term functional outcome following minimally invasive endoscopic intracerebral hemorrhage evacuation. J Neurointerv Surg. 2020;12(5):489-94.

24. Chartrain AG, Kellner CP. A review and comparison of three neuronavigation systems for minimally invasive intracerebral hemorrhage evacuation. J Neurointerv Surg. 2018;10(1):66-74.
25. Barlas O, Karadereler S, Bahar S, et al. Image-guided keyhole evacuation of spontaneous supratentorial intracerebral hemorrhage. Minim Invasive Neurosurg MIN. 2009;52(2):62-8.

26. Keep RF, Hua Y, Xi G. Intracerebral haemorrhage: mechanisms of injury and therapeutic targets. Lancet Neurol. 2012;11(8):720-31.

27. Wu G, Wang L, Hong Z, Mao Y, Hu X. Effects of minimally invasive techniques for evacuation of hematoma in basal ganglia on cortical spinal tract from patients with spontaneous hemorrhage: observed by diffusion tensor imaging. Neurol Res. 2010;32(10):1103-9.

28. Ye Z, Ai X, Hu X, Fang F, You C. Comparison of neuroendoscopic surgery and craniotomy for supratentorial hypertensive intracerebral hemorrhage: a meta-analysis. Medicine. 2017;96(35): 7876 .

29. Eroglu U, Kahilogullari G, Dogan I, et al. Surgical management of supratentorial intracerebral hemorrhages: endoscopic versus open surgery. World Neurosurg. 2018;114:e60-5.

30. Jang SH, Kwak SG, Chang MC. Diabetes does not affect motor recovery after intracerebral hemorrhage. Transl Neurosci. 2020;11(1):277-82.

31. Rindler RS, Allen JW, Barrow JW, Pradilla G, Barrow DL. Neuroimaging of intracerebral hemorrhage. Neurosurgery. 2020;86(5):E414-e423. 